\title{
Identification of magnetosheath mirror modes in Equator-S magnetic field data
}

\author{
E. A. Lucek $^{1}$, M. W. Dunlop ${ }^{1}$, A. Balogh ${ }^{1}$, P. Cargill ${ }^{1}$, W. Baumjohann ${ }^{2}$, E. Georgescu ${ }^{2,4}$, \\ G. Haerendel $^{2}$, K.-H. Fornacon ${ }^{3}$ \\ ${ }^{1}$ The Blackett Laboratory, Imperial College, London SW7 2BZ, UK \\ E-mail: e.lucek@ic.ac.uk \\ ${ }^{2}$ Max-Planck Institute für extraterrestrische Physik, Garching, Germany \\ ${ }^{3}$ Institut für Geophysik und Meteorologie, Technische Universtät Braunschweig, Braunschweig, Germany \\ ${ }^{4}$ Institute of Space Sciences, Bucharest, Romania
}

Received: 10 March 1999 / Revised: 30 June 1999 / Accepted: 20 July 1999

\begin{abstract}
Between December 1997 and March 1998 Equator-S made a number of excursions into the dawnside magnetosheath, over a range of local times between 6:00 and 10:40 LT. Clear mirror-like structures, characterised by compressive fluctuations in $|B|$ on occasion lasting for up to $5 \mathrm{~h}$, were observed during a significant fraction of these orbits. During most of these passes the satellite appeared to remain close to the magnetopause (within 1-2 Re), during sustained compressions of the magnetosphere, and so the characteristics of the mirror structures are used as a diagnostic of magnetosheath structure close to the magnetopause during these orbits. It is found that in the majority of cases mirror-like activity persists, undamped, to within a few minutes of the magnetopause, with no observable ramp in $|B|$, irrespective of the magnetic shear across the boundary. This suggests that any plasma depletion layer is typically of narrow extent or absent at the location of the satellite, at least during the subset of orbits containing strong magnetosheath mirror-mode signatures. Power spectra for the mirror signatures show predominately field aligned power, a well defined shoulder at around 3$10 \times 10^{-2} \mathrm{~Hz}$ and decreasing power at higher frequencies. On occasions the fluctuations are more sinusoidal, leading to peaked spectra instead of a shoulder. In all cases mirror structures are found to lie approximately parallel to the observed magnetopause boundary. There is some indication that the amplitude of the compressional fluctuations tends to be greater closer to the magnetopause. This has not been previously reported in the Earth's magnetosphere, but has been suggested in the case of other planets.
\end{abstract}

Key words. Magnetospheric physics (magnetosheath; plasma waves and instabilities; magnetopause, cusp and boundary layers)

\section{Introduction}

Between December 1997 and March 1998 the Equator-S satellite crossed into the magnetosheath during at least 31 orbits (initially surveyed by Dunlop et al., 1999, and hereafter referred to as paper 1). The magnetosheath observations extend from 06:00 to 10:40 LT and, as the orbit evolves dawnwards, they become biased towards higher solar wind ram pressure $\left(\mathrm{P}_{\mathrm{RAM}}\right)$. With an orbital apogee of $11.5 \mathrm{Re}$, the satellite remained in the magnetosheath for many hours, within 1-2 Re of the magnetopause, during several of the orbits, and was therefore well positioned to observe wave activity in this region of the dawn-side magnetosheath. Examination of the magnetic field data recorded by the MAM experiment (paper 1) showed that approximately $30 \%$ of orbits contained strongly compressional magnetic field signatures in the magnetosheath, often located within a few minutes of the magnetopause. Lucek et al. (1999) (hereafter referred to as paper 2) briefly surveyed these events, showing that they were consistent with mirror structures.

Under conditions of temperature anisotropy (where $T_{\perp}>T_{\|}$), two types of plasma instability can operate which generate low frequency waves (i.e. the wave frequency lies below the proton gyro-frequency $\left(\Omega_{i}\right)$ ). One of these, the ion cyclotron instability, dominates under conditions of high temperature anisotropy (when the parallel proton temperature $\left(T_{\| p}\right)$ is low), and the proton plasma beta $\left(\beta_{p}\right) \sim 1$, and generates transverse electromagnetic ion cyclotron (EMIC) waves through a resonant wave particle interaction. The fastest growing modes are those propagating in a direction parallel to the background magnetic field (i.e. the angle between the wavevector and the field direction $\left(\theta_{k B}\right) \sim 0^{\circ}$, e.g. Schwartz et al., 1996). High frequency EMIC waves $\left(f \sim 0.5-1 \Omega_{i}\right)$ are generally found to be left-hand circularly polarised, but lower frequency waves can be linearly polarised (Anderson et al., 1991; Anderson and Fuselier, 1993). The second instability to operate under conditions of temperature anisotropy is the mirror 
instability. The growth rates of EMIC waves would be expected to dominate those of mirror structures in a purely proton plasma, but the presence of additional cold ion species, such as helium, is thought to suppress the ion cyclotron instability (Price et al., 1986; Gary et al., 1993). Empirically, therefore, the mirror instability dominates under conditions of moderate temperature anisotropy and high beta, and generates strongly compressive (frequently showing $\Delta B / B \sim 1$ ), linearly polarised, non-propagating structures. Within the structures, the plasma and magnetic pressures are anti-correlated. If the plasma just exceeds the condition for stability to mirror waves, then the fastest growing modes are those with $\theta_{k B}$ close to $90^{\circ}$. For larger temperature anisotropy $(>2)$ the growth rate is highest for modes propagating obliquely $\left(\theta_{k B} \sim 60^{\circ}\right)$ (Price et al., 1986). There is also some evidence from simulations to suggest that as mirror structures evolve, they move to longer wavelengths (scale size $\sim 20$ proton gyro-radii) (McKean et al., 1994), and that saturated mirror structures can give rise to larger amplitude magnetic field enhancements (e.g. Schwartz et al., 1996).

In the magnetosheath there are two mechanisms which can be seen to generate a temperature anisotropy where $T_{\perp}>T_{\|}$: gyratory ion motion arising from reflected ions at the bow shock under quasi-perpendicular conditions, and compression of the magnetosheath field close to the magnetopause. Close to the nose of the magnetosphere, for instance, especially under conditions of northward IMF (giving conditions of low magnetic shear at the magnetopause), alignment of the magnetosheath field with the magnetopause arises from transverse IMF orientations (quasi-perpendicular conditions near local noon). Plasma pileup leads to a region of both depleted plasma density and an enhanced magnetic field intensity lying adjacent to the magnetopause (e.g. $\mathrm{Wu}$, 1992). This region, where the temperature anisotropy is high and $\beta$ is moderate, is called the plasma depletion layer (PDL), or sometimes the magnetopause transition layer (e.g. Phan et al., 1994) and is generally found to be unstable to the growth of circularly polarised EMIC waves (e.g. Anderson et al., 1991, 1994; Anderson and Fuselier, 1993; Hubert et al., 1989; Phan et al., 1994); but stable to mirror mode growth. Outside of the PDL the magnetosheath is often found to be unstable to the growth of mirror structures (e.g. Anderson and Fuselier, 1993; Anderson et al., 1994; Phan et al., 1994). These studies show that close to the subsolar point the region identified as a PDL generally lasts for between 10 and 30 min between the magnetopause crossing and the onset of mirror-like activity. The PDL tends to be absent under conditions of radial IMF and/or southward IMF (when transport across the magnetopause is likely to be high). The implied field geometry, however, is most relevant to the equatorial region near local noon, rather than the flanks.

Paper 2 illustrated the typical features of the magnetosheath mirror-like structures in Equator-S data by presenting data from one long interval of mirror-like activity, lasting for about $5 \mathrm{~h}$ and occurring at $\sim 9: 00$ LT. A more comprehensive account of the occurrence and nature of the magnetosheath signatures will be given here. Intervals of mirror-like activity identified to date are listed and a number of selected events are also discussed in some detail. Mirror structures have been identified in spin-averaged data $(\sim 0.67$ vectors $/ \mathrm{s})$ primarily by the occurrence of large amplitude $(\Delta|B| /$ $|B| \sim 0.25-1)$ fluctuations in the magnetic field magnitude, within a low frequency envelope, together with a maximum variance direction (for the de-trended field) which was closely aligned with the background (DC) magnetic field direction. It is expected that clear mirror structures will be identified using these criteria, but marginal cases might be missed without the plasma data for confirmation.

Since the Equator-S magnetosheath data are mainly recorded near the magnetopause, intervals of mirror-like activity are likely to be associated with the magnetic field configuration close to the magnetopause, rather than temperature anisotropy generated at the bow shock. There are a few orbits however, where the magnetosheath data within an hour or so of the magnetopause show no clear evidence for either a PDL or mirror activity, but later in the interval some mirror activity is observed (paper 1). These intervals of mirror activity are documented for completeness, but are more likely to be related to conditions at the bow shock rather than conditions of the magnetosheath close to the observed magnetopause, or large changes in the local magnetopause conditions.

\section{Overview}

As discussed in paper 2, compressive magnetic field signatures consistent with mirror mode structures occur on approximately $30 \%$ of orbits where the satellite entered the magnetosheath and these are shown in Fig. 1. This figure shows an overview, projected onto the $(X, Y)_{\text {GSE }}$ plane, of the locations in LT of the intervals of mirror-like activity (heavy lines) within the Equator-S orbits (dotted lines), and the associated magnetopause crossings (open squares). It is clear from the figure that several of the intervals of mirror activity are long, up to $5 \mathrm{~h}$. The figure also shows cuts through two nominal magnetopause locations (Sibeck et al., 1991) at a height $Z_{\mathrm{GSE}}$ above the $(X, Y)_{\mathrm{GSE}}$ plane which is representative of the crossings, one under conditions of low solar wind pressure $\left(\mathrm{P}_{\mathrm{RAM}} \sim 1.8 \mathrm{nPa}\right)$ and the other for high $\mathrm{P}_{\text {RAM }}(9 \mathrm{nPa})$ (see paper 1). These show that as the spacecraft moved to earlier LT, the magnetopause crossings are biased to higher $\mathrm{P}_{\mathrm{RAM}}$. It is important to note, however, that the magnetopause cuts do not represent actual magnetopause positions at the times of the crossings. Nevertheless, most intervals clearly occur around apogee.

In papers 1 and 2 it was commented that the distinguishing feature of the Equator-S orbits containing clear mirror-like signatures was the large proportion showing mirror activity continuing undamped to within a few minutes of the magnetopause, and the general absence of signatures consistent with a well developed 


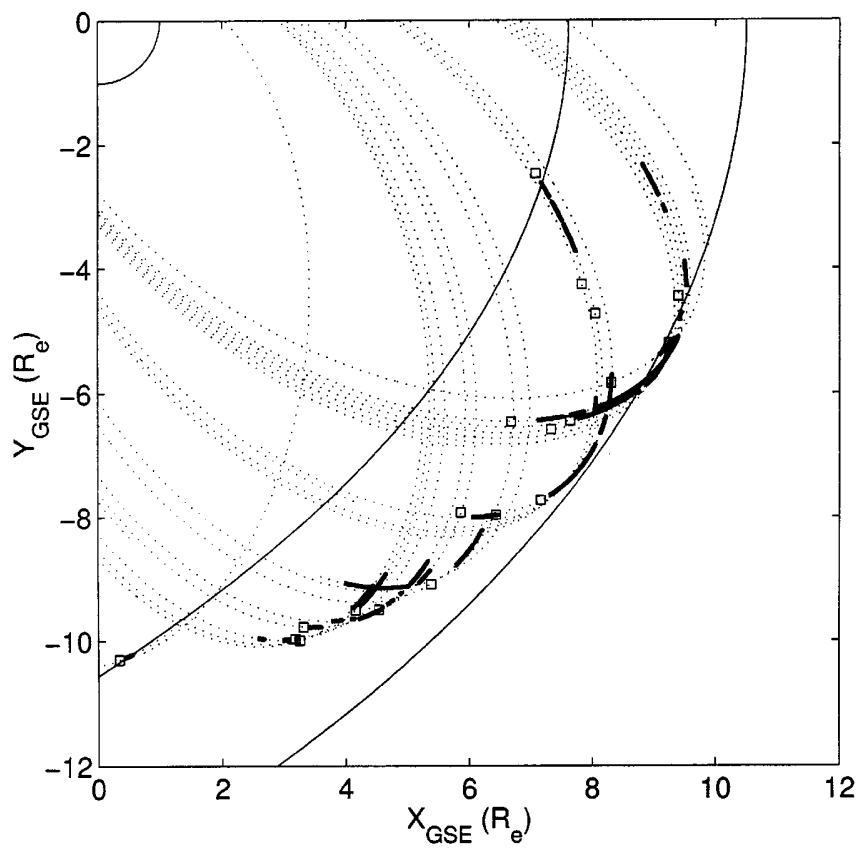

Fig. 1. An overview of the occurrence of mirror-like signatures (solid lines) within Equator-S orbits (dotted lines) and associated magnetopause crossings (open squares). Also shown, for reference are two nominal magnetopause locations, one at high $\mathrm{P}_{\mathrm{RAM}}(9 \mathrm{nPa})$ and one at low $\mathrm{P}_{\mathrm{RAM}}(1.8 \mathrm{nPa})$

PDL. Most Equator-S magnetopause crossings occurred under stable conditions and the magnetic field orientation was generally found to lie in a plane parallel to the magnetopause, consistent with the satellite making a relatively shallow excursion into the magnetosheath. The existence of mirror signatures adjacent to the magnetopause crossing therefore suggests that for most traversals into the magnetosheath a well-developed transition layer, or plasma depletion layer (PDL), was significantly narrower than nearer noon, or absent. There is only one clear example of a signature consistent with a PDL in this subset of orbits. This subset of magnetopause crossings was found to exhibit a range of shear conditions, including one at extremely low shear, suggesting that the occurrence of a PDL is not as strongly dependent on magnetopause shear conditions or upstream IMF orientation at these LT as it is closer to the subsolar point (Anderson and Fuselier, 1993; Phan et al., 1994). It is possible that the general absence of a well-developed PDL in these observations is associated with the dawnside crossing locations (away from local noon) and the Equator-S observations do not necessarily imply the absence of a PDL at all local times. Recent observations made in the dusk flank of the magnetosheath between 15:00 and 17:00 LT (Seon et al., 1999) have not found any evidence for the existence of a PDL.

Each interval of magnetosheath data generally contains several bursts of mirror-like activity, often interrupted by data gaps, but also occasionally isolated instances of mirror structures (Fig. 1, Table 1 also lists the intervals). In the examples which follow more detailed orbit plots are shown on which each individ- ual interval of identified mirror-like activity is indicated over the period where magnetosheath data were taken.

\section{Event analysis}

Currently, plasma data are not routinely available, so that mirror-like signatures have been identified and characterised using only the magnetic field signature. In particular, the characteristic anti-correlation of magnetic and thermal pressures expected within mirror structures cannot be confirmed and no information is available about the local temperature anisotropy or plasma $\beta$. Identification of mirror structures was based primarily on the presence of strong, regular fluctuations in the magnitude of the magnetic field, within a gradually varying envelope of high and low values. In addition, locally de-trended subsets of 60 data points were used to calculate the angle between the local maximum variance direction and the background field direction $\left(\theta_{e B}\right)$. For mirror structures this angle would be expected to remain close to $0^{\circ}$, rising to around $30^{\circ}$ under conditions of large temperature anisotropy (Price et al., 1986). The use of minimum variance analysis means that intervals lasting for less than a few minutes would not be identified. Furthermore, this criterion tends to select intervals showing smaller amplitude fluctuations in field direction present on a stable or slowly varying orientation. Such a signature showing predominantly compressional power is another characteristic of mirror structures. This is best revealed in a plot of the magnetic field vector in polar co-ordinates, as in the examples below. Spin-averaged field measurements $(\sim 0.67$ vectors/s) were used for the majority of the analysis, but some intervals of high resolution data were examined to confirm that there was negligible power at frequencies above that corresponding to the shortest mirror dropouts. It was expected that during intervals of mirror-like activity, the field variations typically would lie approximately parallel to the background field. This magnetic field direction is generally found, for these events, to be parallel to the magnetopause boundary, indicating that the satellite remains relatively close to the magnetopause, and therefore the maximum variance direction of the mirror structures would be expected to lie parallel to the magnetopause. In order to test this, the angle, $\alpha_{e N}$, between the maximum variance direction and the closest magnetopause normal, taken from the analysis presented in paper 1, was monitored. In most cases this angle lay close to $90^{\circ}$.

The event presented in paper 2, December 20, 1997, occurred during two distinct magnetosheath field orientations, corresponding to firstly a high magnetic shear across the magnetopause (observed), and secondly, following a sector boundary crossing, a low shear (inferred). The observations throughout the interval show that the mirror signatures appeared to be unaffected by the sector boundary. Data are presented from five other orbits where Equator-S 
observed mirror-like signatures in the magnetosheath, occurring over the range of LT and for a variety of $\mathrm{P}_{\mathrm{RAM}}$. Two of the examples (December 21, 1997; March 5, 1998) are low- to intermediate-shear crossings; one contains two magnetopause crossings under conditions of exceptionally high $\mathrm{P}_{\mathrm{RAM}}$ : the first is followed by a region consistent with a PDL, and the second shows mirror activity occurring immediately adjacent to it (January 8, 1998), one does not have a clear magnetopause crossing but contains an interval of mirror-like structures which extends throughout the magnetosheath, up to the bow shock (January 21, 1998), and the last is the longest magnetopause crossing interval to be observed which showed changing shear conditions (January 6, 1998). These examples are selected to demonstrate the types of signatures seen and to show that the different observations have many features in common.

\section{December 21, 1998}

Figure 2a shows an overview of the magnetic field data from December 21, 1997. This example was chosen because it is a long interval of mirror-like activity which shows several major changes in characteristics. The

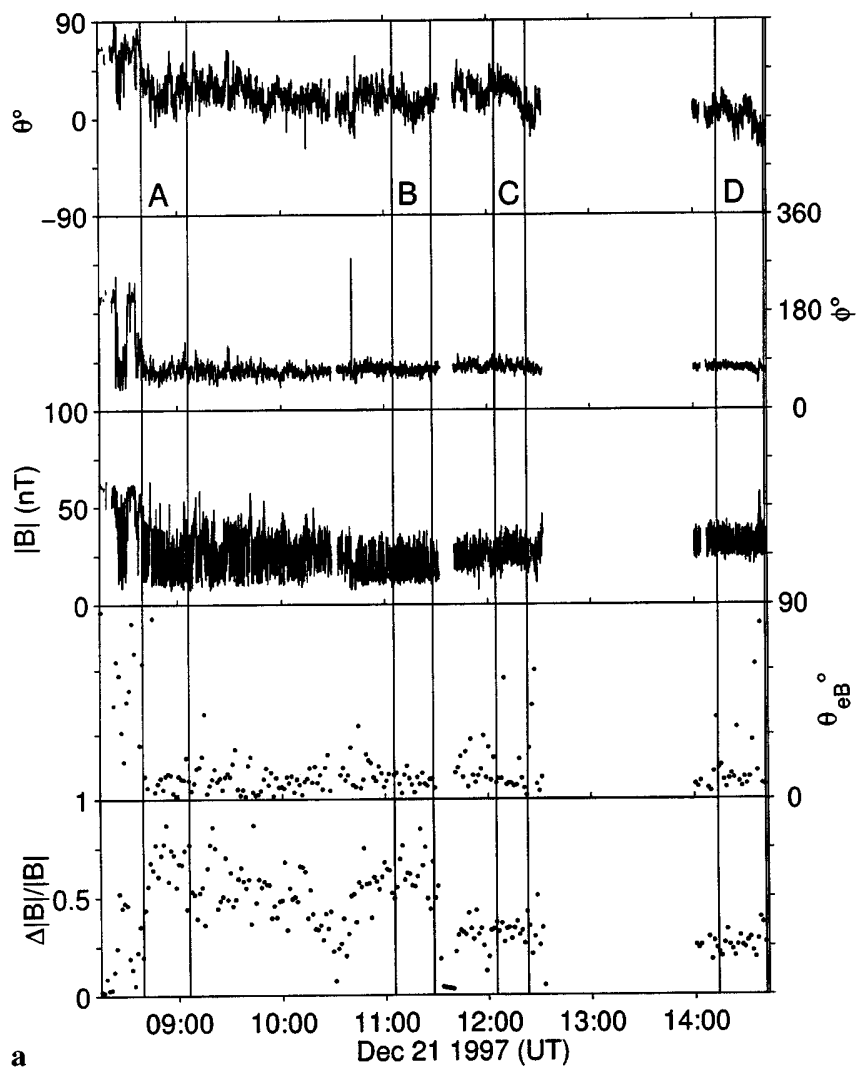

Fig. 2. a An overview of the magnetic field data containing mirrorlike signatures observed on December 21, 1997. The panels show magnetic field elevation $(\theta)$ and longitude $(\phi)$ angles in degrees, magnetic field magnitude $|B|$ in $\mathrm{nT}$, the angle between the maximum variance direction and the mean field direction $\left(\theta_{e B}\right)$ in degrees and $\Delta|B| /|B|$. The magnetic field magnitude data from labelled regions are figure shows the elevation $(\theta)$ and azimuth field $(\phi)$ angles of the magnetic field in GSE co-ordinates, magnetic field magnitude $|B|$, the angle between the maximum variance direction and the average field direction calculated using variance analysis on locally de-trended subsets of 60 data points $\left(\theta_{e B}\right)$, and $\Delta|B| /|B|$. Positive $\theta$ indicates a northward pointing field, and $\phi$ is positive duskwards.

There are three magnetopause crossings close together at the beginning of the interval shown in the plot, at 08:25, 08:31 and 08:36 UT. Mirror-like structures occur during the short interval of sheath observations between the first two crossings, and resume immediately following the third. During this orbit, mirror-like signatures were observed for $\sim 5 \mathrm{~h}$. There appears to be an evolution in the characteristics of the signatures with time from the major magnetopause crossing. Immediately following the last magnetopause crossing the mirror-like signatures have large $\Delta|B| /|B|$. The evolution of $\Delta|B| /|B|$ is not smooth, but in general $\Delta|B| /|B|$ tends to become lower as time elapses after the magnetopause crossing. Although this trend is not always present, it may represent an evolution of mirror-like activity with distance from the magnetopause but changes in the local magnetopause position as a result of changing $\mathrm{P}_{\mathrm{RAM}}$ during the interval would complicate the signature.

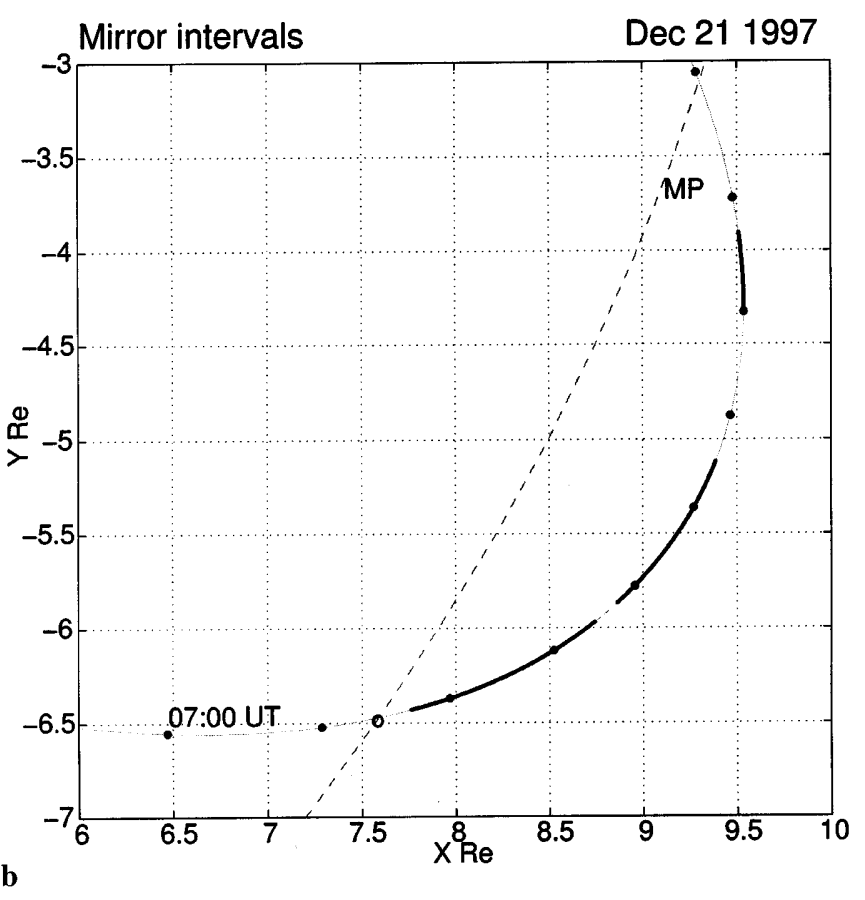

shown in Fig. 3a. b Orbit segment projected onto the $(\mathrm{X}-\mathrm{Y})_{\mathrm{GSE}}$ plane showing intervals of mirror-like activity (thick lines) during the orbit of December 21, 1997, (fine line). Hourly intervals along the orbit track are indicated by solid circles. The magnetopause (dashed line) corresponds to the fitted surface for the crossing as described in the text 
Throughout the interval the magnetic field orientation is consistent with it lying parallel to the magnetopause boundary, suggesting that the satellite remained relatively close to the magnetopause. A similar pattern was observed in the magnetosheath data from December 20, 1997.

Figure $2 \mathrm{~b}$ shows intervals of mirror-like signatures, positively identified by the magnetic field criteria detailed earlier in this section, emphasised by the thick lines, plotted over the orbit track (thin line). Also plotted are hour marks (solid circles) along the orbit from the universal time indicated, the key magnetopause crossing (open circle) and a cut through the particular model magnetopause surface which corresponds to the observed crossing position (Dunlop et al., this issue). The latter indicates the expected relation to the magnetopause, at the $\mathrm{Z}_{\mathrm{GSE}}$ co-ordinate of the crossing (which ranges from $\sim 3-4.8 \mathrm{Re}$ above the $(\mathrm{X}, \mathrm{Y})_{\mathrm{GSE}}$ plane), strictly only at the time of the crossing. The mirror signatures cover nearly $7 \mathrm{~h}$, ending at the end of the data coverage, and with the breaks representing only data gaps. The gap between the magnetopause crossing and the start of the mirror interval is small $(\sim 0.2 \mathrm{Re})$, and in fact contains a partial re-entry into the magnetosphere, confirming that the magnetopause remains at the position indicated, or moves outward slightly, and that mirror signatures start immediately adjacent to the magnetopause. Data coverage for this event stopped before the satellite reentered the magnetosphere.

Figure 3 shows the four intervals of mirror-like activity indicated on Fig. 2a by the labels A, B, C and

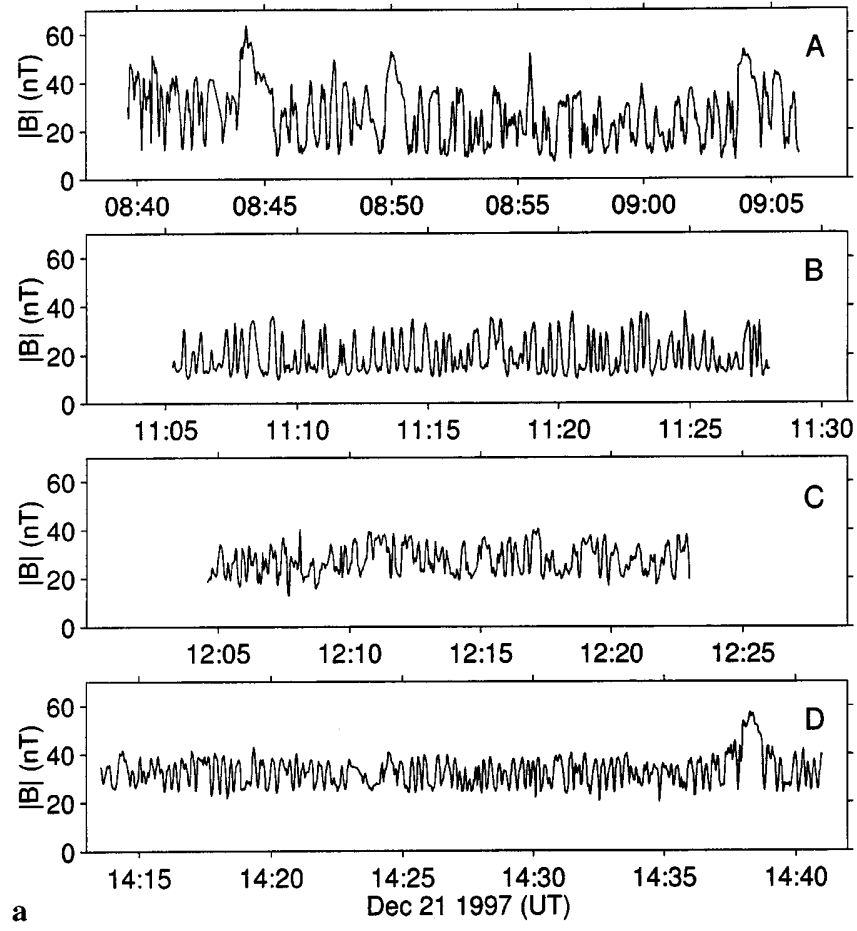

Fig. 3. a The four panels show intervals of magnetic field magnitude data from December 21, 1997, each on the same scales and b the corresponding power spectra of the magnetic field magnitude for each
D. Their field magnitudes on the same scales are shown in Fig. 3a, together with the corresponding power spectral densities for the field magnitude (i.e. compressional power only) in Fig. 3b. The first interval (A) shows mirror-like signatures soon after the magnetopause crossing. They are strongly compressive and are interrupted by larger amplitude, larger scale structures. It has been suggested that this type of feature can evolve from mirror structures which persist after saturation (e.g. Schwartz et al., 1996). Such structures are common features in the Equator-S observations. The power spectrum for this interval has the typical fall off in power at high frequencies, starting at $\sim 2 \times 10^{-2} \mathrm{~Hz}$ in this case. There is a change in gradient at this point, but instead of the low frequency portion of the spectrum being flat, as is usual, the larger scale structures contribute additional power at lower frequencies. The second interval (B) shows mirror-like structures of smaller amplitude, and smaller scale size, and represents the most regular appearance. It exhibits the most stable values for $\theta_{e B}$, which remain between $\sim 5-15^{\circ}$. The power spectrum shows the typical decrease in power at high frequencies, above $\sim 5 \times 10^{-2} \mathrm{~Hz}$, the higher frequency reflecting the smaller scale size of the structures, and a slight excess of power at around $5 \times 10^{-2} \mathrm{~Hz}$, arising from the more regular nature of the structures. The third interval (C) shows smaller amplitude mirror-like structures showing a typical power spectrum. There is no evident evolution of the minimum scale size of the structures between intervals 2 and 3 . The fourth interval (D) shows mirror-like structures of the same amplitude as for $\mathrm{C}$
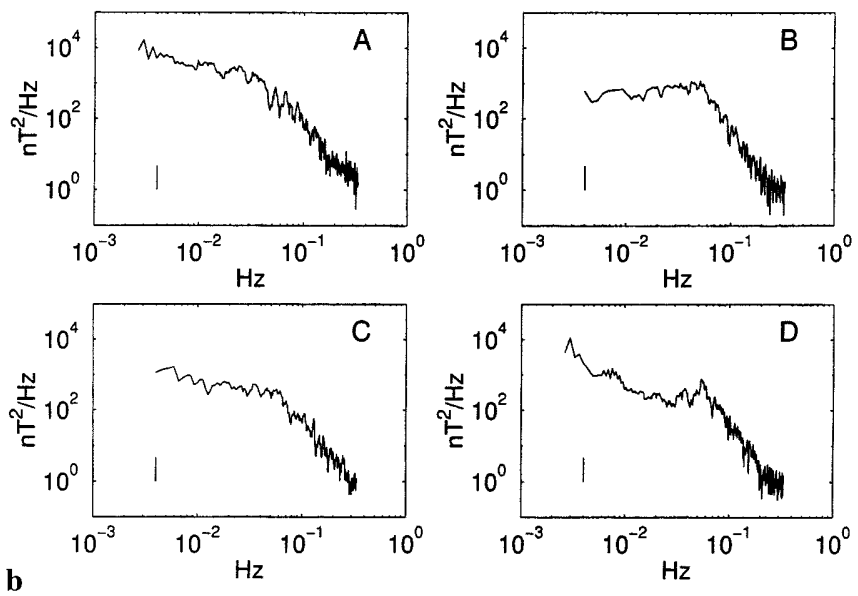

of the intervals in a. The $90 \%$ confidence limit is shown in the bottom left hand corner 
but of regular, quasi-sinusoidal appearance and smaller scale size. The power spectrum shows a well-defined peak at $\sim 6 \times 10^{-2} \mathrm{~Hz}$.

Quasi-sinusoidal structures are seen frequently in the Equator-S data, especially during longer intervals of mirror-like activity. Previous studies have also reported quasi-sinusoidal mirror-like structures (e.g. Bavassano Cattaneo et al., 1998; Tsurutani et al., 1982; Phan et al., 1994). Although the characteristic shoulder in the power spectra lies well below the Nyquist frequency of spin-averaged data, it is instructive to view the times series at higher resolution. Overlays of the spin-averaged and high resolution $(64 \mathrm{~Hz})$ data are shown in Fig. 4 for two intervals. Figure 4 a shows the interval just before $\mathrm{A}$, after the magnetopause crossing but outside the mirror interval. Figure $4 \mathrm{~b}$ shows an interval within B. It is clear that within B the high resolution data shows only very low amplitude deviations from the spin resolution data, otherwise following the mirror structures closely. In contrast, Fig. 4a demonstrates that outside the intervals identified as mirror signatures, there can be significant power at higher frequencies. Figure $4 \mathrm{c}$, shows $\phi$ for the same interval as Fig. 4a, and demonstrates that these higher frequency fluctuations are not dominated by the compressional power as in the mirror cases.

Figure 5a summarises the ordering of the magnetic field variations with respect to the magnetopause boundary normal. The top panel shows a scatter plot of the field angles $\theta$ (latitude) and $\phi$ (longitude) in GSE co-ordinates, recorded between 08:10 and 08:42 UT during the magnetopause crossing on December 21, 1997, together with curves representing the plane defined by the minimum variance direction, and a fit to the scatter. The minimum variance direction can be interpreted as the normal to the magnetopause boundary. The bottom panel shows $\alpha_{B N}$, the elevation angle of the field with respect to the maximum variance plane calculated using the data shown in the top panel. A value $\alpha_{B N} \sim 0$ indicates that the field vector lies in a plane parallel to the magnetopause boundary. The systematic deviation of $\alpha_{B N}$ away from 0 could be explained either by a flaring of the magnetic field as the satellite moves deeper into the magnetosheath, a changing magnetosheath field or the change in local magnetopause normal arising from curvature of the magnetopause as the satellite moves along the boundary. Figure $5 \mathrm{~b}$ demonstrates the ordering within the fluctuations by the MVA magnetopause boundary normal. The top panel shows the magnetic field magnitude for reference and the lower panel shows the angle between the maximum variance direction and the magnetopause normal $\left(\alpha_{e N}\right)$. If the maximum variance direction of the mirror structures lies parallel to the magnetopause then this angle would be expected to be close to $90^{\circ}$. At the start of the interval of mirror-like activity $\alpha_{e N}$ is $\sim 90^{\circ}$ with a scatter of $\sim 20^{\circ}$. Later in the interval, after $\sim 11: 00 \mathrm{UT}$, the deviation between the maximum variance direction and the normal increases, while the overview plot shows that $\theta_{e B}$ remains approximately constant.
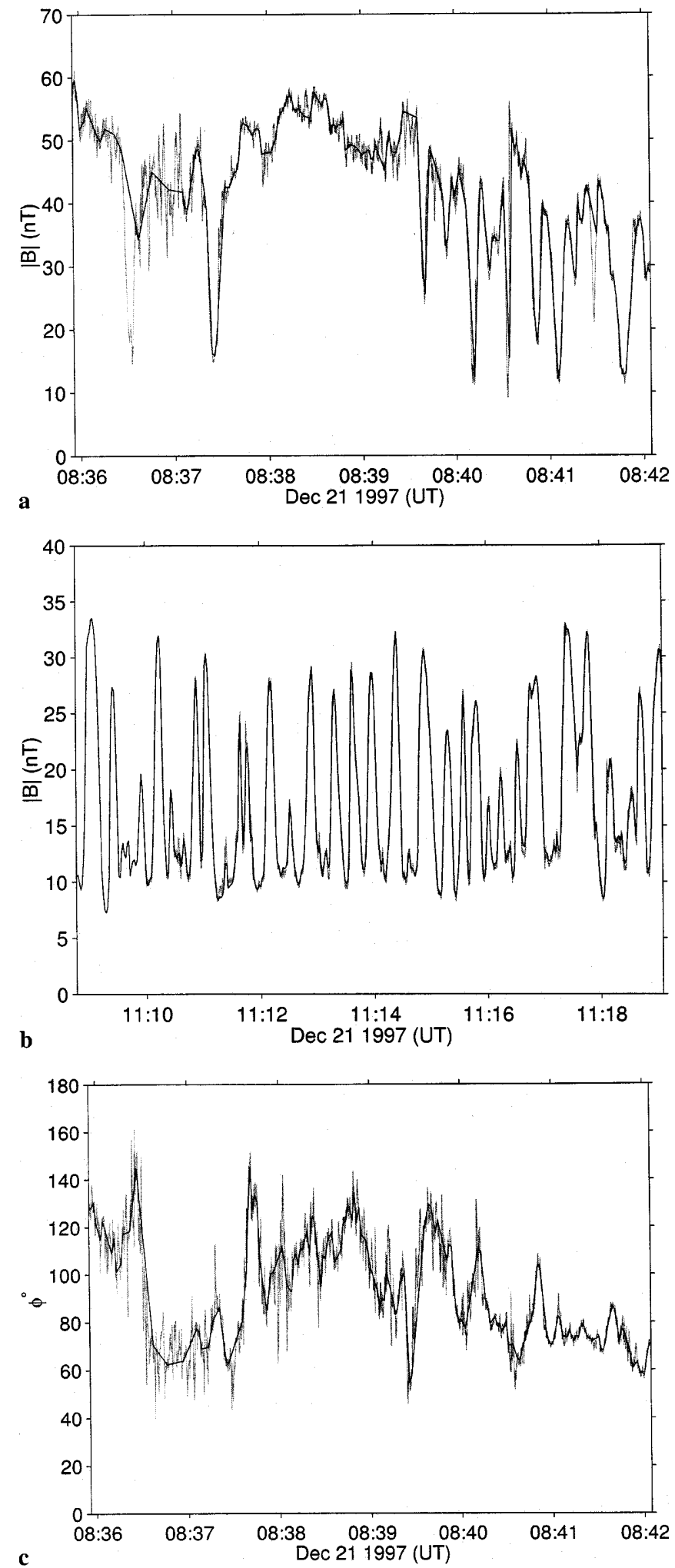

Fig. 4a-c. Overlay of high resolution and spin-averaged magnetic field magnitude data for a the interval next to the magnetopause, just before the start of interval $A, \mathbf{b}$ a portion of interval $B$ and $\mathbf{c}$ the corresponding plot for the field angle $\phi$ for the same interval as shown in $\mathbf{a}$ 

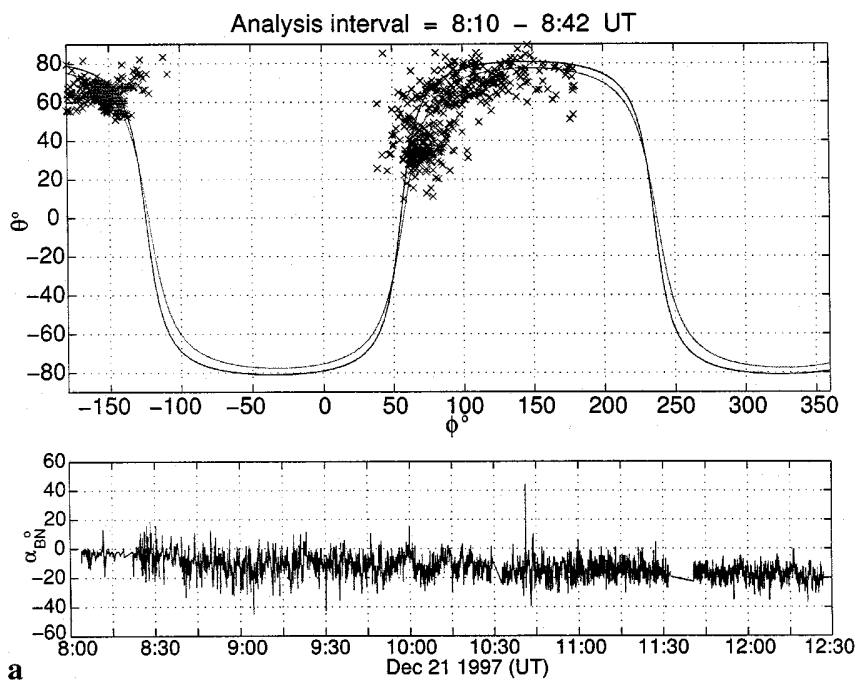

Fig. 5. a The top panel shows a scatter plot of the field angles $\theta$ (latitude) and $\phi$ (longitude) in GSE co-ordinates, between 08:10 and 08:42 UT during the magnetopause crossing on December 21, 1997, together with curves representing the plane defined by the minimum variance direction, and a fit to the scatter. The minimum variance direction can be interpreted as the normal to the magnetopause boundary. The bottom panel shows $\alpha_{B N}$, the elevation angle of the field

\section{January 6, 1998}

The data recorded on January 6, 1998, showed a number of excursions into the magnetosheath, and on one occasion the spacecraft appeared to skim the magnetopause boundary for some time, before briefly entering the magnetosphere and then returning to the magnetosheath proper again. Figure 6 a shows an overview of the magnetic field data on January 6, 1998 and Fig. 6b shows the January 6, 1998, event projected onto the satellite orbit. Here, the magnetopause moves outwards after the first crossing (MP1) and then appears to return, finally re-crossing the spacecraft at the position marked MP2. Times of these identified magnetopause crossings (paper 1) are indicated on Fig. 6a by vertical dashed lines. Mirror-like signatures occur before and up to the crossing at 04:36 UT although $\Delta|B| /|B|$ decreases as the magnetopause approaches (Fig. 7a, panel B). No mirror-like signatures are observed during the following two short excursions into the magnetosheath, but resume approximately $5 \mathrm{~min}$ after the crossing at 05:58 UT. During the next two hours there is gradual rotation of $\theta$ to the magnetospheric orientation, and during the latter part of this interval $\Delta|B| /|B|$ decreases again as the mirror-like dips in the field become more widely separated (Fig. 7a, panel B). During this time, therefore, it appears that the spacecraft gradually approaches the magnetopause, followed by a sharp traversal of the magnetopause back into the magnetosheath at 08:22 UT. Mirror-like signatures, appearing as well spaced dips and indicated on Fig. 6a by a dotted line, follow immediately (Fig. 7a, panel C) and continue, interrupted by a data gap, until the final entry into the magnetosphere at 10:24 UT (MP3) when the magnetopause has almost the same location as for MP1.

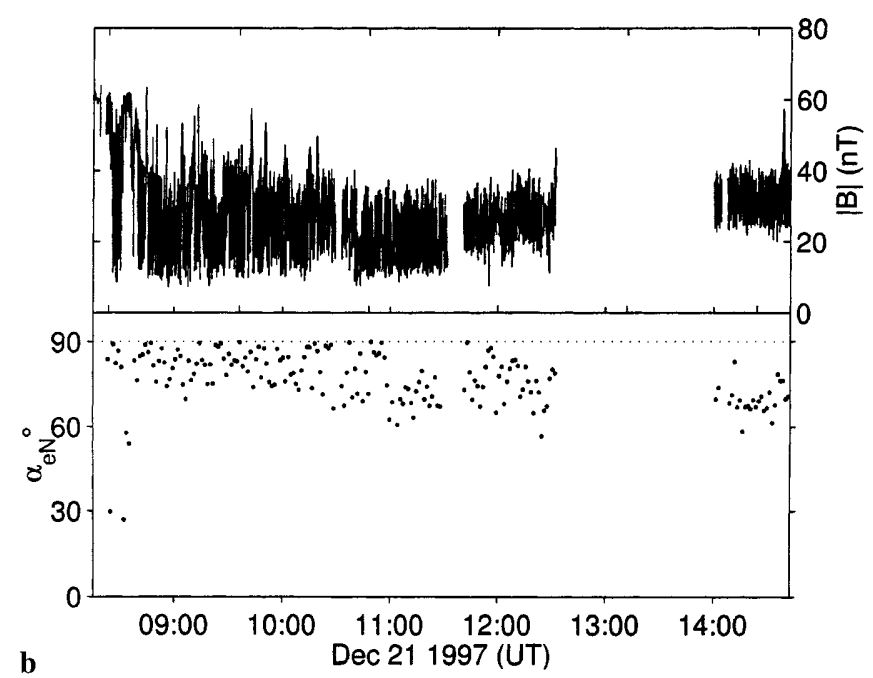

with respect to the maximum variance plane calculated using the data shown in the top panel. b The corresponding analysis within the interval of mirror activity where the top panel shows $|B|$ and the bottom panel shows the angle between the maximum variance direction calculated using locally detrended subsets of 60 data points and the magnetopause normal direction calculated using boundary normal analysis $\left(\alpha_{e N}\right)$ (paper 1)

Results are available from boundary normal analysis of three of the magnetopause crossings (see paper 1), at 04:36, 05:52 and 10:24 UT. Each of the three intervals shown in Fig. 7a are well ordered by the closest magnetopause crossing, indicating that fluctuations lie approximately parallel to the magnetopause boundary throughout (Fig. 7b).

\section{January 8, 1998}

Figure $8 \mathrm{a}$ shows the magnetic field recorded on January 8, 1998, and Fig. 8b shows the orbit plot. The magnetosphere was in a compressed state under conditions of high solar wind ram pressure during this event. The spacecraft is already past apogee at the time of the first exit from the magnetosphere (MP1). The magnetopause is clearly moving inwards at this time and only recrosses the spacecraft at 10:53 UT, corresponding to the end of the mirror interval shown, and makes a final reentry into the magnetosphere at $\sim 11: 03$ (MP2). The data show a significant change in the character of the mirror-like structures between about 10:19 and 10:23 UT when there is a transition between dips in a high field region to peaks in a low field region (marked with a dashed line on Fig. 8b). The later mirror-like structures also have a more sinusoidal form which gives rise to a broad peak in the power spectrum. Analysis of these and other mirror-like structures shows that the peaks and dips do not have distinct spectral characteristics.

This orbit is the only example which shows a clear tailing off of the compressional signatures closer to the magnetopause, and in fact both crossings exhibit low shear $\left(\sim 60^{\circ}\right)$. The field magnitude between the magnetopause (at 09:25 UT) and about 09:40 UT shows a 


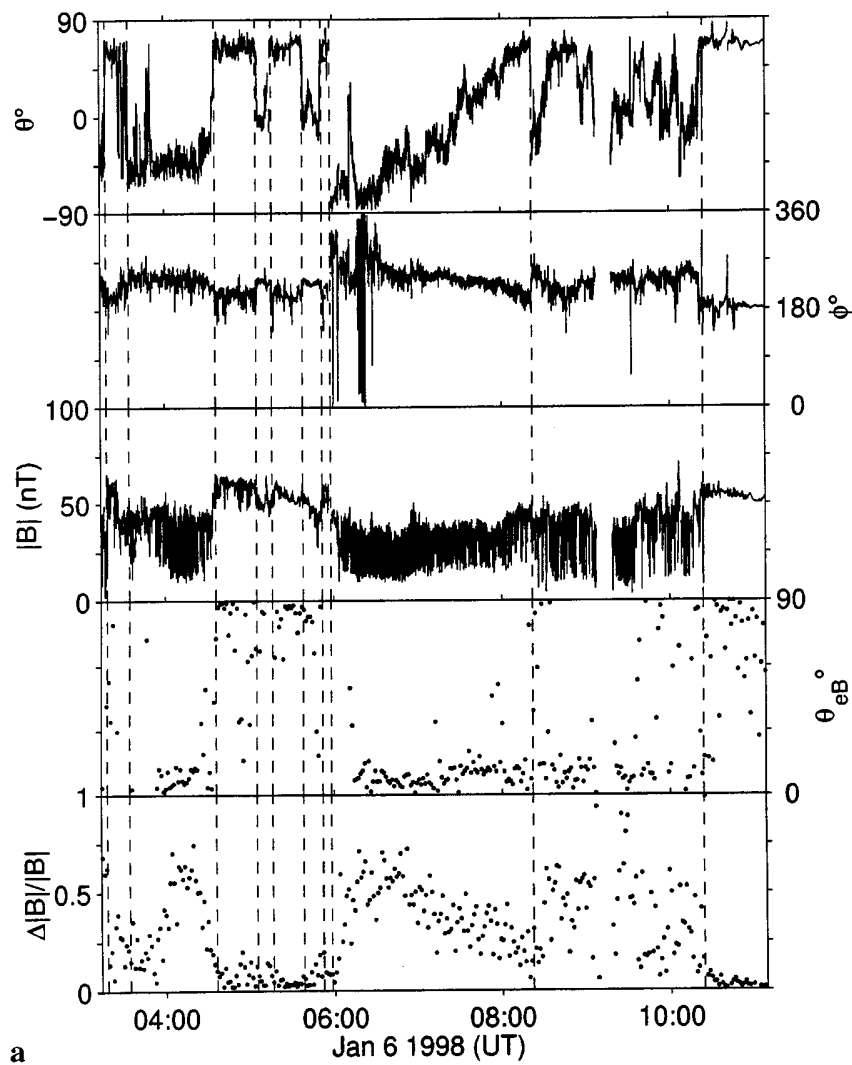

Fig. 6. a An overview of the magnetic field data recorded on January 6, 1998, in the same format as Fig. 2. The panels show field elevation $(\theta)$ and longitude $(\phi)$ angles in degrees, field magnitude $|B|$ in nT, the angle between the maximum variance direction and the mean field

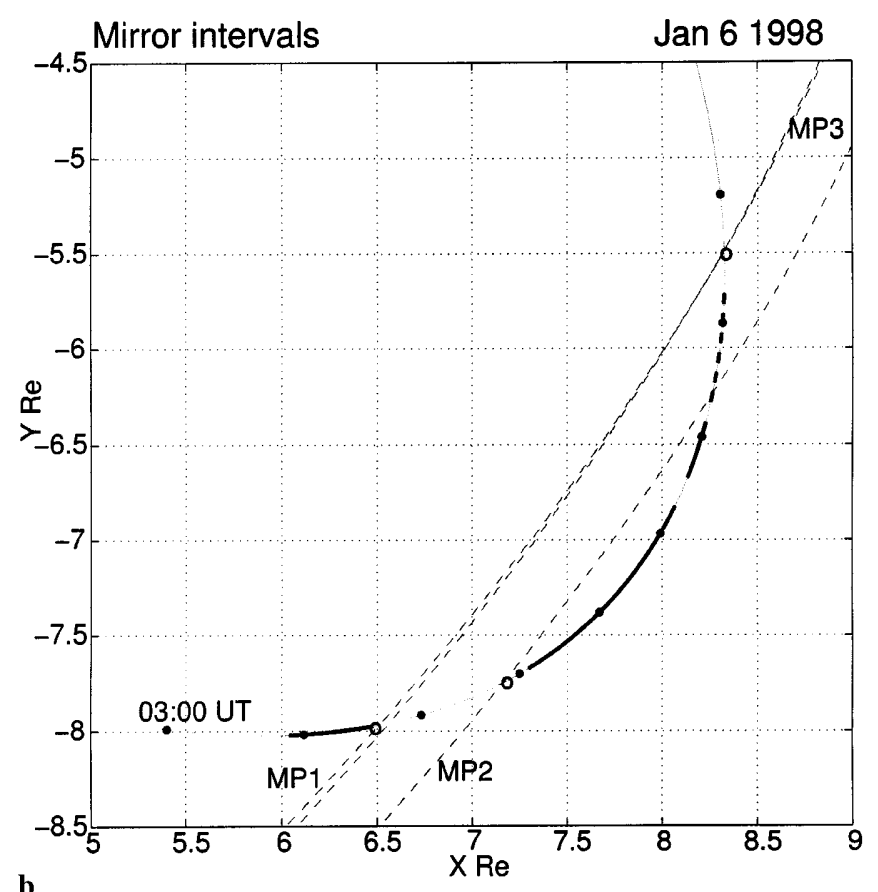

direction $\left(\theta_{e B}\right)$ in degrees and $\Delta|B| /|B|$. The vertical dashed lines indicate times of identified magnetopause crossings. b Intervals of mirror activity projected onto the orbit segment for January 6, 1998, in the form described for Fig. $2 b$ ramp in the field, typical of the PDL (Anderson and Fuselier, 1993; Phan et al., 1994). The high resolution data during this interval show significant fluctuations in the field at higher frequencies than covered by the spin resolution data. Figure 9 shows a close up of the two components of the high frequency fluctuations which lie perpendicular to the background field direction. The fluctuations are circularly polarised and therefore consistent with EMIC waves.

The high frequency fluctuations reduce in amplitude as the region of compressional signatures is approached, so that the traces match in the 'mirror' region (similar to the trace in Fig. 4b). This characteristic is in contrast to the later encounter (at 11:02 UT), which shows large amplitude fluctuations right up to the magnetopause (first crossing at $\sim 10: 50$ UT). The crossings occur at $\sim 10: 00$ and 10:40 LT, respectively, the latter representing the crossing location closest to local noon. The difference between the two encounters is primarily the degree of magnetospheric compression at the magnetopause. Although the early encounter shows an enhanced field magnitude, the later encounter shows a higher magnetic compression which is associated with an extremely high solar wind ram pressure (Dunlop et al., this issue).

\section{January 21, 1998}

Figure 10a shows magnetic field data from the orbit on January 21, 1998. There is an interval of mirror-like activity lasting for nearly $2 \mathrm{~h}$ at the beginning of the interval. This orbit was omitted from the original study of mirror-like signatures because it does not show a clear magnetopause crossing. The orbit is unique within the Equator-S data set, however, in that the dynamic pressure conditions were extremely high $(\sim 14-15 \mathrm{nPa})$ and the satellite passed into the solar wind later in the orbit, traversing the bow shock. The IMF data recorded upstream suggest that the bow shock would be quasiperpendicular in nature at the time (Haerendel, private communication, 1999). The intervals marked with vertical lines in Fig. 10a contain intervals of transverse wave activity which are likely to be situated in the foreshock. The magnetopause boundary shown in the orbit plot (Fig. 10b) is estimated using the observed upstream solar wind ram pressure and suggests that the mirror structures start approximately $2.5 \mathrm{Re}$ from the magnetopause. The mirror structures then persist throughout the magnetosheath, gradually reducing in amplitude with increasing time (Fig. 10a), until the satellite crosses into the solar wind. Figure $10 \mathrm{~b}$ also shows that the local time of the satellite is almost 

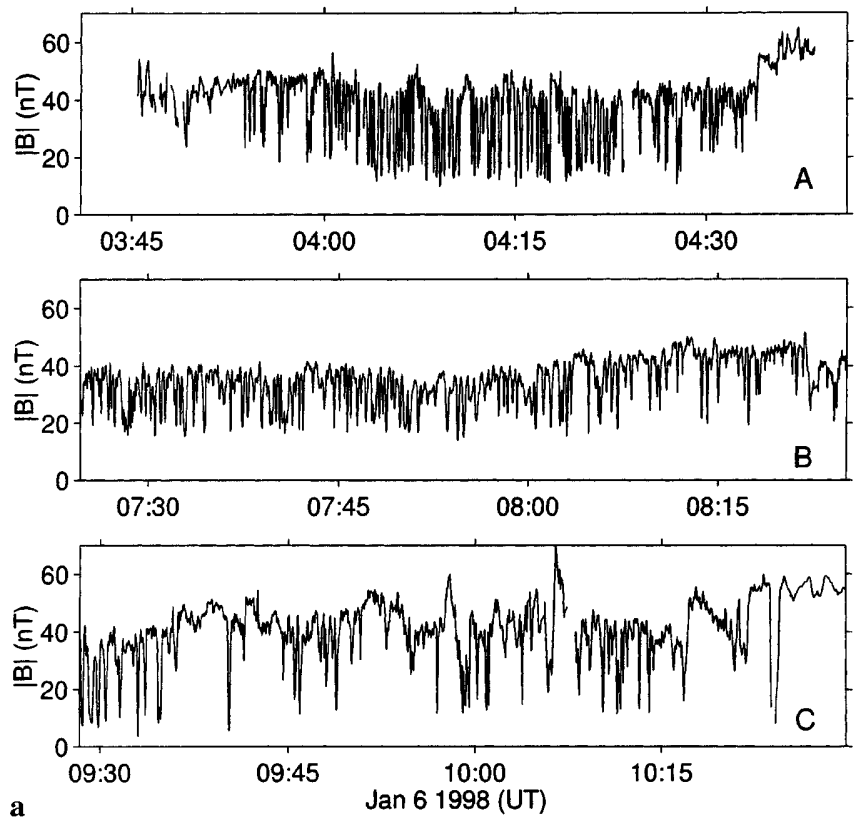

Fig. 7. a Three intervals of mirror-like activity on January 6, 1998. The first occurs just before a magnetopause crossing, the second occurs during a gradual evolution of $\theta$ towards a magnetospheric orientation, which preceded a sharp return to the magnetosheath, and the third occurs before the final magnetopause crossing observed on
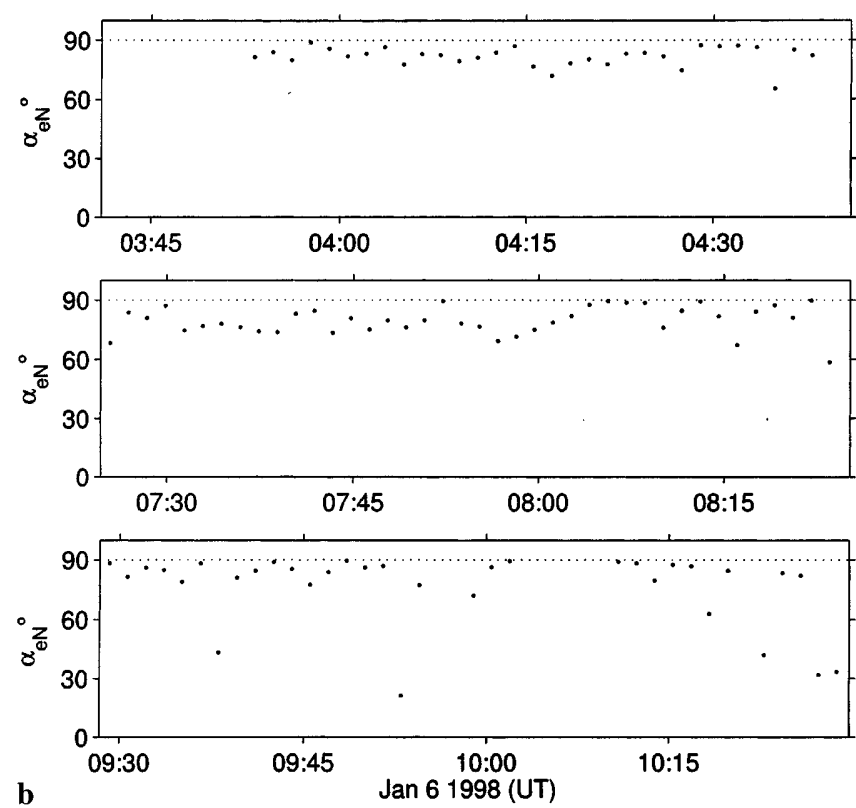

that orbit. b This shows the ordering of the fluctuations during the three intervals. Each panel shows the angle between the maximum variance direction and the closest magnetopause normal $\left(\alpha_{e N}\right)$. Values close to $90^{\circ}$ indicate that the fluctuations lie in a plane parallel to the observed magnetopause boundary

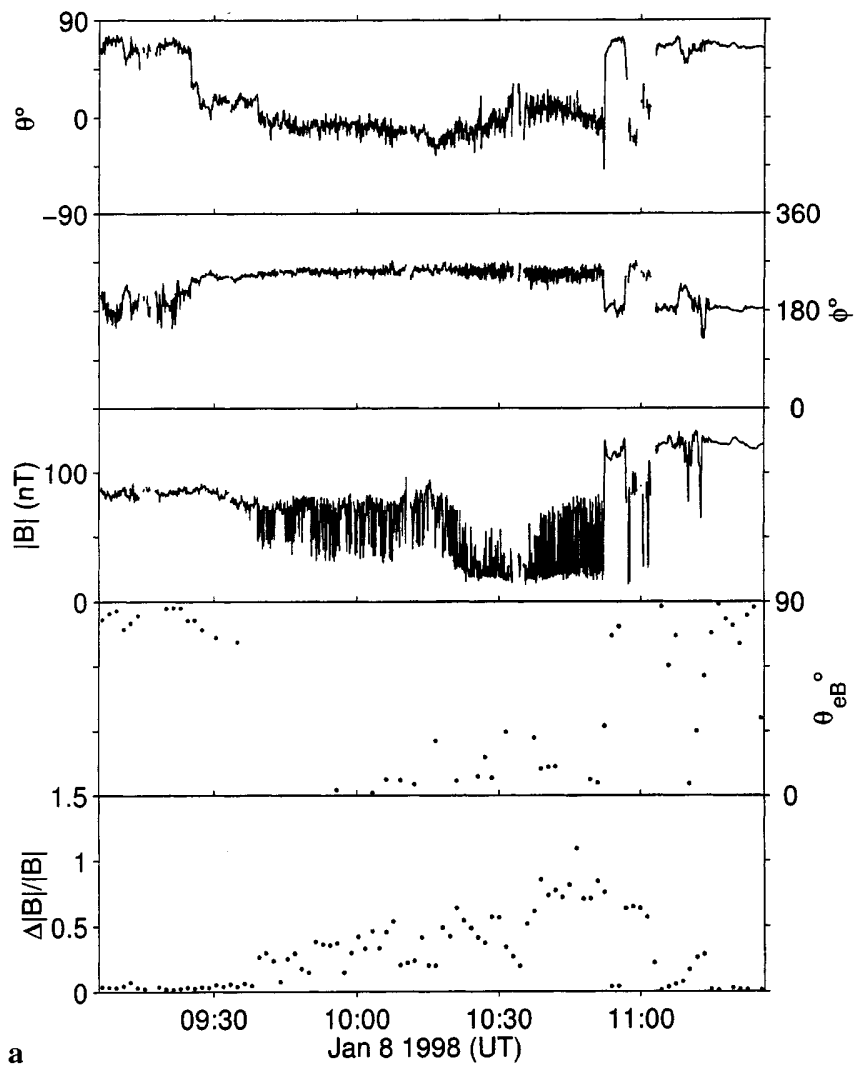

Fig. 8. a An overview of the mirror-like signatures observed on January 8, 1998. There is a sharp change in the characteristics of the structures where they change from dips to peaks. b Intervals of mirror

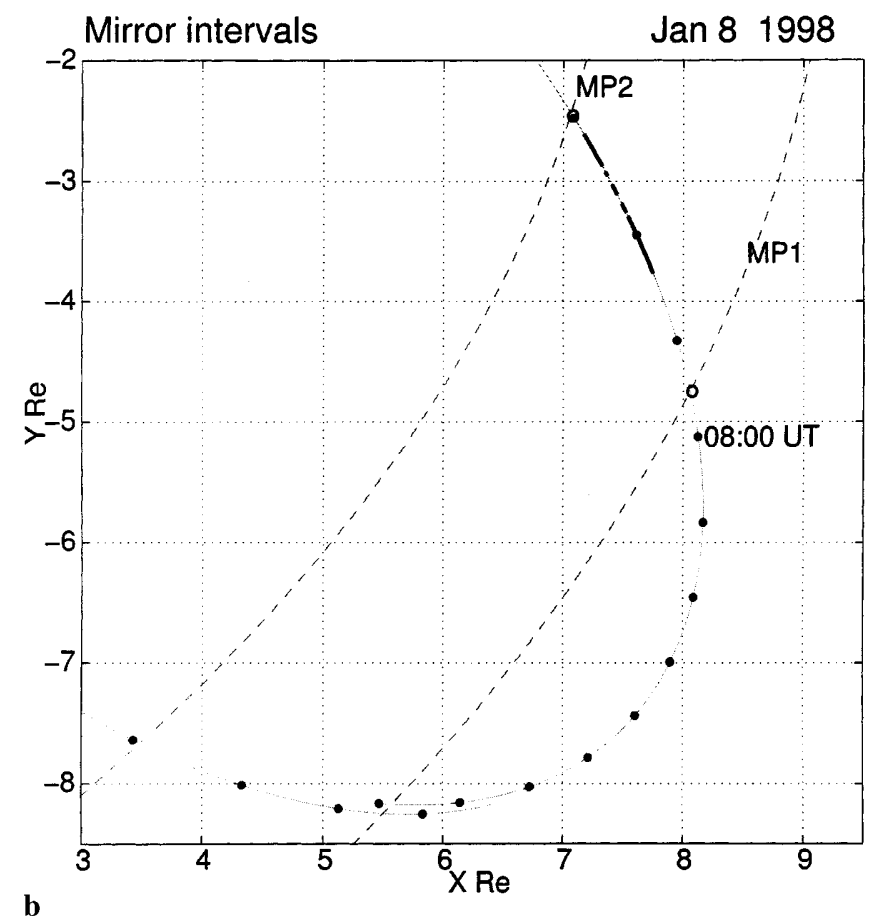

activity projected onto the orbit segment for January 8, 1998, in the form described for Fig. $2 b$ 


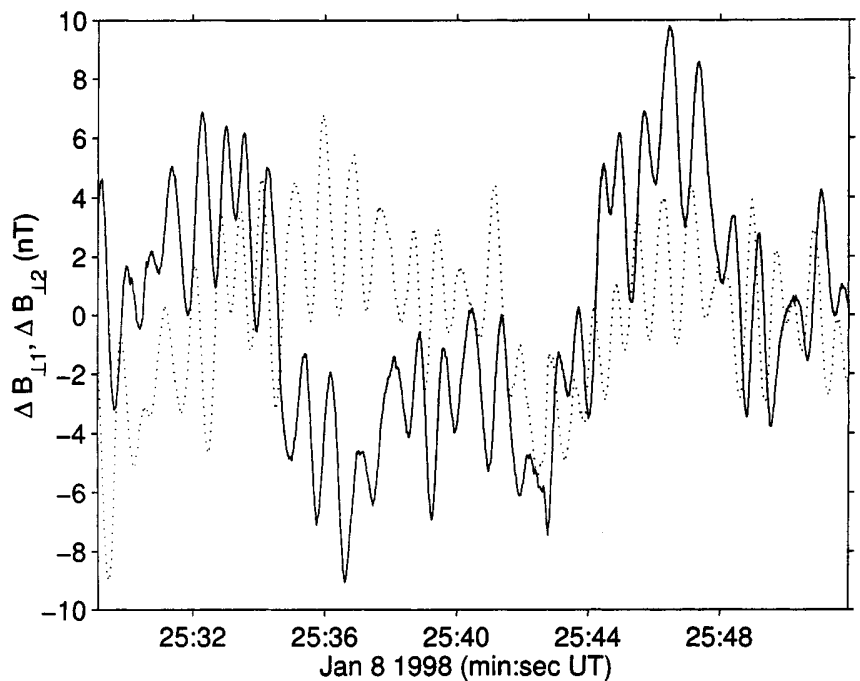

Fig. 9. The two components of the magnetic field perpendicular to the background field direction on January 8, 1998. The fluctuations are circularly polarised

constant during the observations of mirror activity. The mirror-like structures at the beginning of the plot have a maximum field direction which lies at approximately $10^{\circ}$ to the background field direction, as is usual. Later in the interval, at around 21:51 UT this angle rises to $\sim 30^{\circ}$.

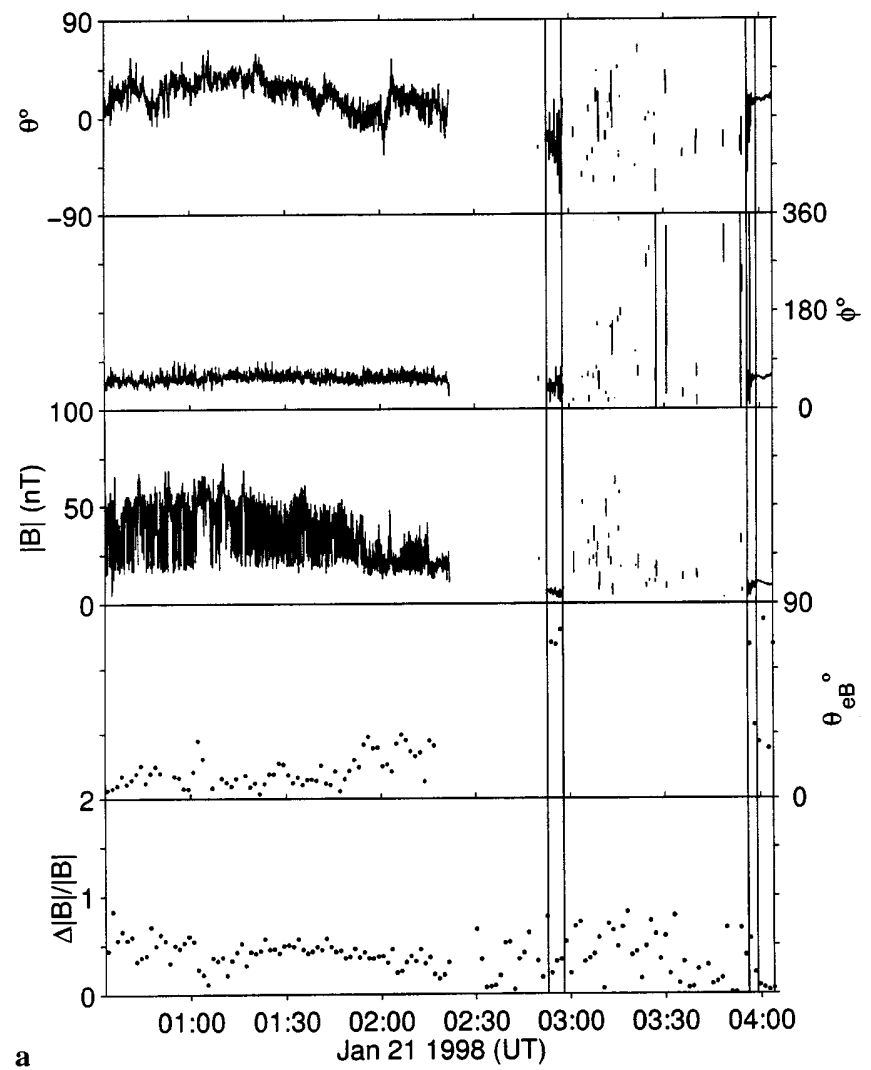

Fig. 10. a An overview of the waves seen on January 21, 1998. The panels show field elevation $(\theta)$ and longitude $(\phi)$ angles in degrees, field magnitude $|B|$ in $\mathrm{nT}$, the angle between the maximum variance direction and the mean field direction $\left(\theta_{e B}\right)$ in degrees and $\Delta|B| /|B|$. Mirror-like signatures occur during approximately half the interval.
This is consistent with $T_{\perp} / T_{\|}>2$ (Schwartz et al., 1996). This behaviour is also seen in the data from February 1, 1998. The field direction is stable throughout the magnetosheath as might be expected downstream of a quasi-perpendicular shock, under conditions where the solar wind ram pressure is unusually high. Using minimum variance analysis within the mirror structures shows that their orientation changes little during the interval.

\section{March 5, 1998}

This event was chosen because it was the last interval of mirror-like activity to be seen, during the final orbit which crossed into the magnetosheath, at the earliest local time. It also contains the only example of a nearly zero shear magnetopause crossing. The interval of mirror-like activity is relatively short, lasting for about 15 min (Fig. 11a) but it is very well bounded by two clear magnetopause crossings at 21:58 UT and 22:14:30 UT. Figure $11 \mathrm{~b}$ shows the orbit plot for this interval. The magnetopause only just reaches the position of the spacecraft, which then subsequently skirts the boundary. It is clear from the panels showing $\theta$ and $\phi$ (Fig. 11a) that both crossings show very low magnetic shear across the boundary. The change in $\phi$ is of the order of $20^{\circ}$ and

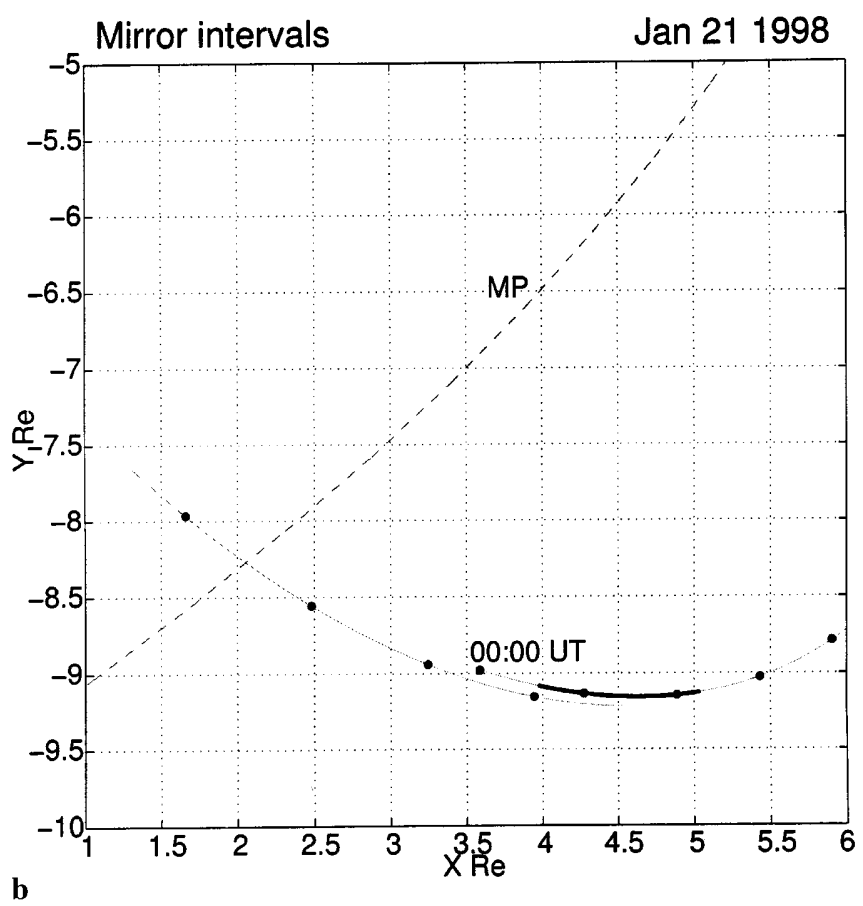

The regions indicated by the vertical lines contain transverse, circularly polarised waves likely to be located within the foreshock. b Intervals of mirror activity projected onto the orbit segment for January 21, 1998, in the form described for Fig. 2b 


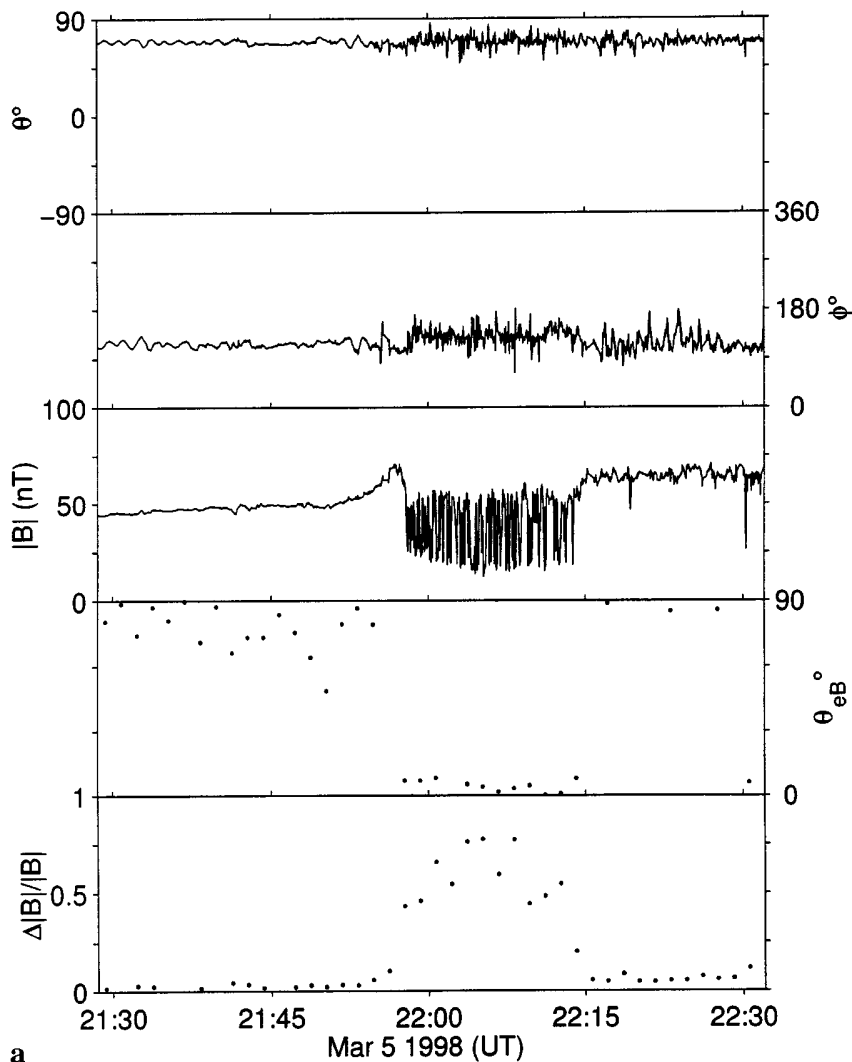

Fig. 11. a Overview of the magnetosheath observations made on March 5, 1998. The panels show field elevation $(\theta)$ and longitude $(\phi)$ angles in degrees, field magnitude $|B|$ in $\mathrm{nT}$, the angle between the maximum variance direction and the mean field direction $\left(\theta_{e B}\right)$ in

the change in $\theta$ just a few degrees. The fluctuations are well ordered by the boundary normal calculated for the first magnetopause crossing. During the interval there is a tendency for the mirror-like structures to become more widely spaced.

\section{Discussion and summary}

In the absence of plasma data, this study has concentrated on the characteristics of the magnetic signatures of mirror-like structures. Fluctuations which satisfy the criteria used here for identification as mirror-like signatures are those which show large amplitude compressive fluctuations within a relatively slowly varying envelope and have a maximum variance direction which is close to the background field direction. The spectral signatures and ordering by closest magnetopause normal were also examined for these intervals. The spectral signature of the mirror-like fluctuations was most typically flat up to a frequency between $3-8 \times 10^{-2} \mathrm{~Hz}$, where a shoulder occurred in the power spectral density of the field magnitude, followed by a rapid fall off with increasing frequency. The position of the shoulder represents approximately the smallest scale at which the field oscillates within the high and low field envelope. Higher frequency oscillations are often of significantly

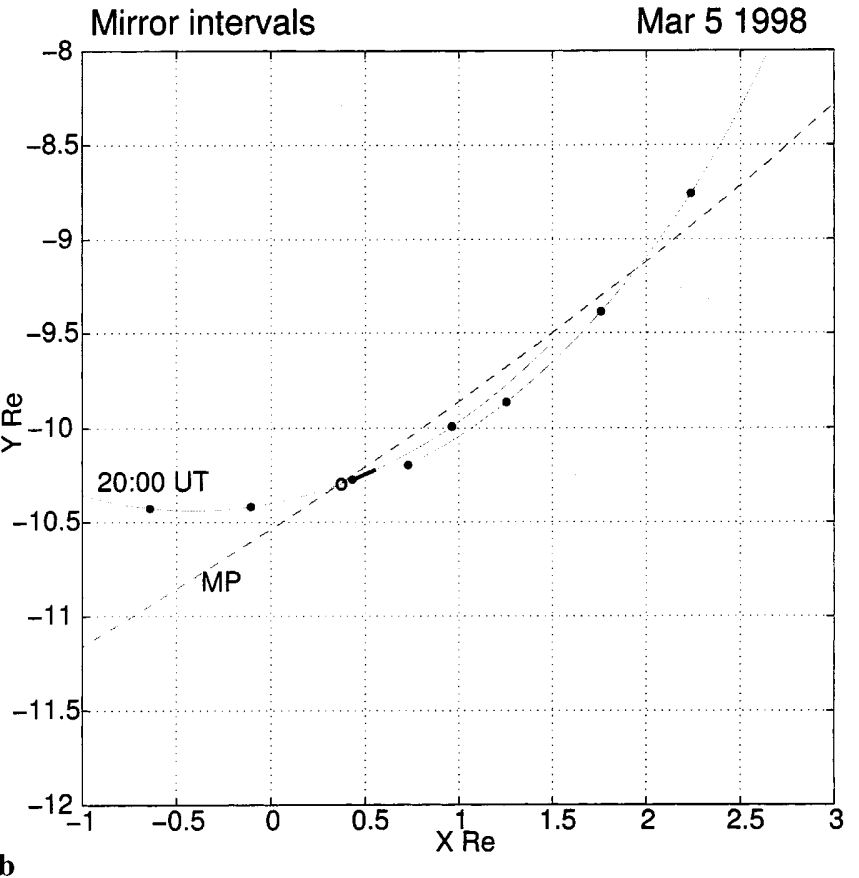

degrees and $\Delta|B| /|B|$. Large amplitude mirror-like signatures occur between the two low shear magnetopause crossings. b Intervals of mirror activity projected onto the orbit segment for March 5, 1998, in the form described for Fig. $2 b$

smaller amplitude. Near sinusoidal wave forms are quite frequently observed (e.g. Fig. 3a, panels B and D). The power spectra for these waves tend to have an excess of power at the frequency of the shoulder (e.g. Fig. 3b, panels B and D). Mirror-like waves frequently have a less stable envelope (e.g. Fig. 3a, panel C), additional angular variations, or are interrupted by larger scale magnetic structures (e.g. Fig. 3a, panel A). In these cases the power spectra show an excess of power at low frequencies (e.g. Fig. 3b, panels A and C). The power spectrum appears to be insensitive to whether the mirror-like structures occur as dips or peaks (e.g. Fig. 8a). As yet the distributions of magnitudes, spatial sizes and frequency of occurrence of the individual dips and peaks within each interval of activity have not been investigated. For this each mirror-like dip or peak would have to be identified, although the lack of velocity data means that the physical size cannot be calculated, or related to the ion gyro-radius.

The ordering of the mirror-like structures was examined in two ways. First the direction of maximum variance, representing the direction within which the magnetic field varied most greatly, was related to the background field direction. One criterion for the identification of mirror-like signatures was that this angle should be lower than $30^{\circ}$, but occasions when the angle had low scatter, but remained at around $30^{\circ}$ are 
consistent with regions of increased temperature anisotropy. Intervals exhibiting this type of behaviour were relatively rare, with only two clear examples in the data set. Secondly the ordering of the magnetic field fluctuations with respect to the nearest magnetopause boundary crossing was examined. By calculating the angle between the maximum variance direction and the magnetopause normal direction, it was confirmed that all mirror-like structures were ordered by the magnetopause boundary and that the maximum variance direction approximately lay at right angles to the magnetopause normal. This is consistent with the structures lying parallel to the magnetopause boundary and the satellite remaining close to the magnetopause where the magnetic field would be typically expected to be aligned with the boundary. On a few occasions, however, there was a systematic discrepancy between the magnetopause boundary plane and the plane within which the mirror structures by (e.g. the latter part of the interval shown in Fig. 5). On these occasions $\alpha_{e N}$ is rarely $90^{\circ}$, but instead is offset from $90^{\circ}$ by up to approximately $25^{\circ}$. This pattern is consistent with the fluctuations lying in a plane which is at a small angle to the previously observed magnetopause. This may represent temporal evolution of the boundary orientation, the flaring of the magnetic field as the satellite moved deeper into the magnetosheath, or the curvature of the magnetopause as the satellite moves along the boundary. The observations from January 21, 1998, have been included because the satellite traversed the whole magnetosheath at nearly constant local time. They are therefore complementary to the other events where only shallow excursions are likely to have occurred, often across a large range in LT. During this orbit the mirror structures show no drift in orientation. This may be explained by the conditions of high solar wind ram pressure at the time, and the orientation of the IMF being consistent with the bow shock having a quasiperpendicular orientation.

There is some evidence to suggest that the size and magnitude of the mirror-like structures increases as the magnetopause is approached. In some of the early observations when the satellite spent up to $6 \mathrm{~h}$ in the magnetosheath there are occurrences of large amplitude compressive structures close to the magnetopause, which have a shoulder in their power spectrum at slightly lower frequencies than those structures occurring later in the interval, which are likely to be further from the magnetopause. In addition, during the orbit when the satellite crossed the bow shock into the solar wind, the amplitude of the mirror structures was higher close to the magnetopause, and lower close to the bow shock. This result is not well supported by other observations made in the Earth's magnetosheath, but previous observations of mirror structures between the bow shock and magnetopause at Saturn (Bavassano Cattaneo et al., 1998) showed a similar behaviour until the satellite entered the magnetosheath transition layer. Although observations are available over a range of local time, there is no obvious dependence of the characteristics of the mirror-like signatures on LT. This may be a consequence of the criteria used for their identification.

Compared with previous observations closer to local noon, an unexpectedly large proportion of orbits which contain clear mirror-like structures show mirror-activity continuing to within a few minutes of, if not adjacent to, magnetopause crossings, under a variety of shear conditions. This suggests that the PDL is likely to be greatly reduced in extent or absent at the location of the spacecraft. Observations near the sub-solar point show that the PDL is least well-developed or absent under conditions of poor alignment between the boundary and the IMF, or large shear leading to substantial momentum transfer across the boundary. Since the magnetosheath intervals recorded by Equator-S occur under conditions of both high and low shear, it is suggested that the lack of a well-developed PDL is a function of the satellite location on the dawn flank and does not necessarily imply that the PDL is poorly developed throughout the magnetosheath. A future study will include more detailed analysis of the short intervals between magnetopause crossings and the onset of mirror activity, in order to establish whether the PDL is narrow or absent at these times. Recent observations made by the Geotail satellite, in the dusk flank of the magnetosheath, found no evidence of a PDL at late LT.

Acknowledgements. Analysis of Equator-S data at Imperial College is supported by PPARC. The Equator-S mission was made possible through grant 500C 94024 by the German Space Agency, (DARA/DLR).

Topical Editor K.-H. Glassmeier thanks J.A. Slavin and another referee for their help in evaluating this paper.

\section{Appendix}

Table 1 summarises the occurrence of mirror-like structures during 13 Equator-S orbits. It is not a definitive list, but contains intervals where the field behaviour satisfied the criteria outlined earlier. The first column gives the date of each orbit. Columns two and three give the time in UT and LT respectively, of the major magnetopause crossing in each orbit, relevant to the observed mirror-like features. For one orbit, that of January 6, 1998, there were two major magnetopause crossings close to mirror-like structures. Column four gives the observed magnetic compression of the magnetosphere, related to a nominal model magnetosphere. For these mirror events there is no strong dependence of the magnetosphere compression on the LT of the magnetopause crossing, except for the two crossings at the earliest local times which also have the highest compression factors. Columns 4 and 5 indicate the magnetic shear observed at the magnetopause crossing. The crossings are classified as low, intermediate or high shear, and column 5 gives the observed angle of rotation of the magnetic field vector across the boundary, taken from Dunlop et al., (this issue). Where there is a clear change in field orientation in the magnetosheath for 
Table 1. Intervals of mirror mode occurrence. For a full description, see text

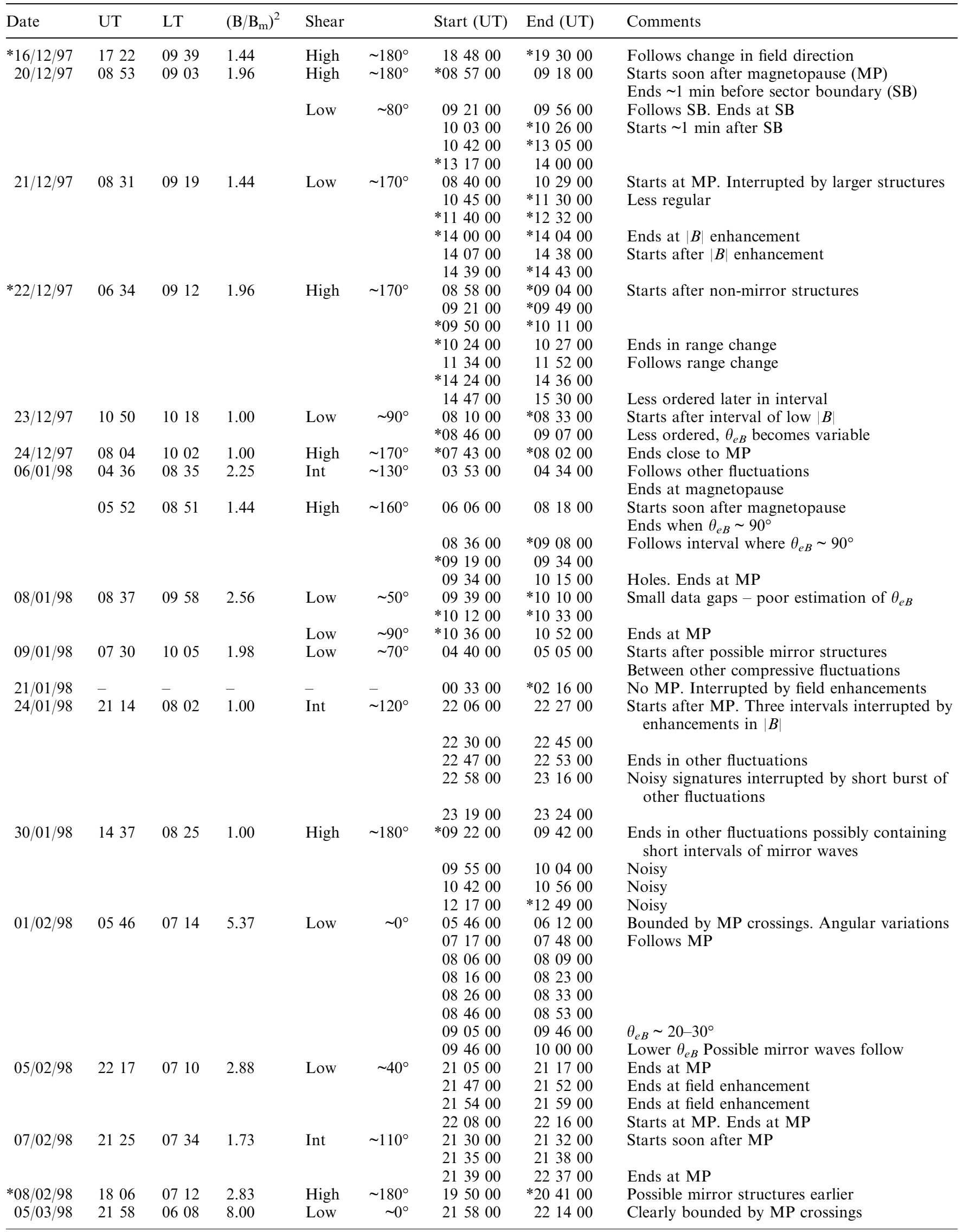


example on December 20, 1997, at the sector boundary, the shear relevant to the mirror-like structures is inferred. Columns 6 and 7 give the start and end times of identified mirror-like structures in UT. Start and end times bounded by data gaps are indicated by an asterisk. These occur much more often during early orbits where there were almost solely long intervals of very clear mirror-like activity. The later orbits tended to have noisier signatures and so mirror-like activity, as defined here, was more frequently bounded by other fluctuations which did not satisfy the criteria. The last column contains comments on the intervals of mirror-like activity covering aspects such as sector boundaries occurring within the interval; the occurrence of larger scale magnetic field magnitude enhancements; proximity of the mirror-like signatures to magnetopause crossings or strong characteristics such as the occurrence of holes instead of peaks.

As described in the Introduction, the Equator-S orbit is such that the satellite typically remains relatively close to the magnetopause, and most intervals of mirror activity listed in Table 1 appear to be connected with the local magnetopause crossing. There are a small number of orbits where the data recorded within an hour or so of the magnetopause show no clear evidence of either a PDL, or mirror activity, but at some time later in the orbit some mirror activity is observed. It is possible that the occurrence of these intervals is related to conditions at the bow shock rather than conditions close to the magnetopause, and occur only rarely as a result of the limited data coverage and the satellite generally remaining close to the magnetopause. Alternatively the mirror activity may arise much later in the orbit as a result of changing magnetopause conditions. These intervals are included for completeness, but they are marked by an asterisk on the date of the orbit.

\section{References}

Anderson, B. J., and S. A. Fuselier, Magnetic pulsations from 0.1 to 4.0 Hz and associated plasma properties in the Earth's subsolar magnetosheath and plasma depletion layer, J. Geophys. Res., 98, 1461-1479, 1993.
Anderson, B. J., S. A. Fuselier, and D. Murr, Electromagnetic ion cyclotron waves observed in the plasma depletion layer, Geophys. Res. Lett., 18, 1955-1958, 1991.

Anderson, B. J., S. A. Fuselier, S. P. Gary, and R. E. Denton, Magnetic spectral signatures in the Earth's magnetosheath and plasma depletion layer, J. Geophys. Res., 99, 5877-5891, 1994.

Bavassano Cattaneo, M. B., C. Basile, G. Moreno, and J. D. Richardson, Evolution of mirror structures in the magnetosheath of Saturn from the bow shock to the magnetopause, $J$. Geophys. Res., 103, 11 961-11 972, 1998.

Dunlop, M. W., A. Balogh, W. Baumjohann, G, Haerendel, K.-H. Fonacon, and E. Georgescu, Dawnside magnetopause observed by the Equator-S magnetic field experiment: identification and survey of crossings, J. Geophys. Res. Brief Rep., in press, 1999.

Gary, S. P., S. A. Fuselier, B. J. Anderson, Ion anisotropy instabilities in the magnetosheath, J. Geophys. Res., 98, 14811488, 1993.

Hubert, D., C. C. Harvey, and C. T. Russell., Observations of magnetohydrodynamic modes in the Earth's magnetosheath at 0600 LT, J. Geophys. Res., 94, 17 305-17 309, 1989.

Lucek, E. A., M. W. Dunlop, A. Balogh, P. Cargill, W. Baumjohann, E. Georgescu, G. Haerendel, and K.-H. Fornacon, Mirror mode structures observed in the dawn-side magnetosheath by Equator-S, Geophys. Res. Lett., in press, 1999.

McKean, M. E., D. Winske, and S. P. Gary, Two-dimensional simulations of ion anisotropy instabilities in the magnetosheath, J. Geophys. Res., 99, 11 141-11 153, 1994.

Phan, T.-D., G. Paschmann, W. Baumjohann, N. Sckopke, and H. Lühr, The magnetosheath region adjacent to the dayside magnetopause: AMPTE/IRM observations, J. Geophys. Res., 99, 121-141, 1994.

Price, C. P., D. W. Swift, and L. -C. Lee, Numerical simulations of nonoscillatory mirror waves at the Earth's magnetosheath, $J$. Geophys. Res., 91, 101-112, 1986.

Seon, J., S. M. Park, K. W. Min, L. A. Frank, W. R. Paterson, and K. W. Ogilvie, Observations of density fluctuations in Earth's magnetosheath with Geotail and Wind spacecraft, Geophys. Res. Lett., 26, 959-962, 1999.

Sibeck, D. G., R. E. Lopez, and E. C. Roelof, Solar wind control of the magnetopause shape, location and motion, J. Geophys. Res., 96, 5489-5495, 1991.

Schwartz, S. J., D. Burgess, and J. J. Moses, Low-frequency waves in the Earth's magnetosheath: present status, Ann. Geophysicae, 14, 1134-1150, 1996.

Tsurutani, B. T., E. J. Smith, R. R. Anderson, K. W. Ogilvie, J. D. Scudder, D. N. Baker, and S. J. Bame, Lion roars and nonoscillatory drift mirror waves in the magnetosheath, $J$. Geophys. Res., 87, 6060-6072, 1982.

Wu, C. C., MHD flow past a obstacle: Large-scale flow in the magnetosheath, Geophys. Res. Lett., 19, 87-90, 1992. 